\title{
Element Distribution in Novel Hedgehog-Like Magnetic Nanostructures Studied by Cs-Corrected STEM-EELS and Uncorrected STEM-XEDS using SDD-Technology
}

\author{
M. Falke ****, A. Mogilatenko**, W. Neumann**, C. Brombacher***, H. Rohrmann****, \\ M. Kratzer****, M. Albrecht***, A. Bleloch*****, R. Terborg*, R. Kroemer*, and M. Rohde* \\ * Bruker-AXS Microanalysis GmbH, Berlin, Germany \\ ** Humboldt University zu Berlin, Germany \\ *** Institute of Physics, University of Technology Chemnitz, Germany \\ **** Data Storage, OC Oerlikon-Balzers AG, Balzers, Liechtenstein \\ ***** superSTEM Daresbury, The University of Liverpool, UK
}

An EDS (energy dispersive x-ray spectroscopy) system incorporating a liquid nitrogen free XFlash 5030 SDD detector covering a solid angle of $0.12 \mathrm{sr}$ on a conventional TEM/STEM was used to complement atomic resolution HAADF images from a NION MarkII Cs-corrected dedicated STEM and the elemental maps achieved using Gatan EELS on the Cs-corrected instrument [1]. The element distribution in the active magnetic layers of novel magnetic nano-structures was characterised and the results were used to interpret their magnetic switching behaviour and support micromagnetic simulations of the necessary energy barrier.

In modern magnetic recording materials the 'superparamagnetic effect' has become a show stopper for higher storage densities. Patterned media [2], comprising two-dimensional arrays of nanostructures with high magnetic anisotropy, are one of the concepts that might provide the required areal density in future magnetic recording devices. Novel hedgehog-like magnetic nanostructures (Fig. 1.) were created by depositing subsequently a seed layer and a $\mathrm{CoPtCr}-\mathrm{SiO}_{2}$ alloy onto two-dimensional arrays of self-assembled $\mathrm{SiO}_{2}$ nano-spheres. We found that by tuning the growth conditions single magnetic domain nano-caps with enhanced magnetic coercitivity fields can be fabricated.

Electron transparent cross sections of the samples with $50 \mathrm{~nm}$ particle size were prepared by tripod polishing and a short ion-milling step. EELS spectrum imaging was used to study the distribution of Cobalt, Chromium, Ruthenium and Oxygen in the deposited nano-structured system (Fig. 2). EDS was used to confirm the distribution of Ru and Platinum (Fig. 3.). The microscopy data reveal that the magnetic caps consist of a closed textured Co-containing film on top of which columnar Cocontaining nano-crystals grow with their growth axes perpendicular to the sphere surface, thus forming a hedgehog-like structure. EELS spectrum imaging and HAADF in Cs-corrected STEM show, that these magnetic nano-columns grow directly from and coupled to crystallites of the closed textured film below, but are separated from each other otherwise by a Chromium and Oxygen containing nm-layer. The inclination of the magnetic nano-crystals provides the easier magnetic switching. The coupling to the closed textured Co-Pt layer underneath is responsible for a strong magnetic coupling throughout the cap of one nano-sphere enabling the switching of single spheres.

\section{References}

[1] O. L. Krivanek, N. Dellby, and A. R. Lupini, Ultramicrocopy 78, (1999) 1.

[2] M. Albrecht et al., Nat. Mater. 4 (2005) 203. 

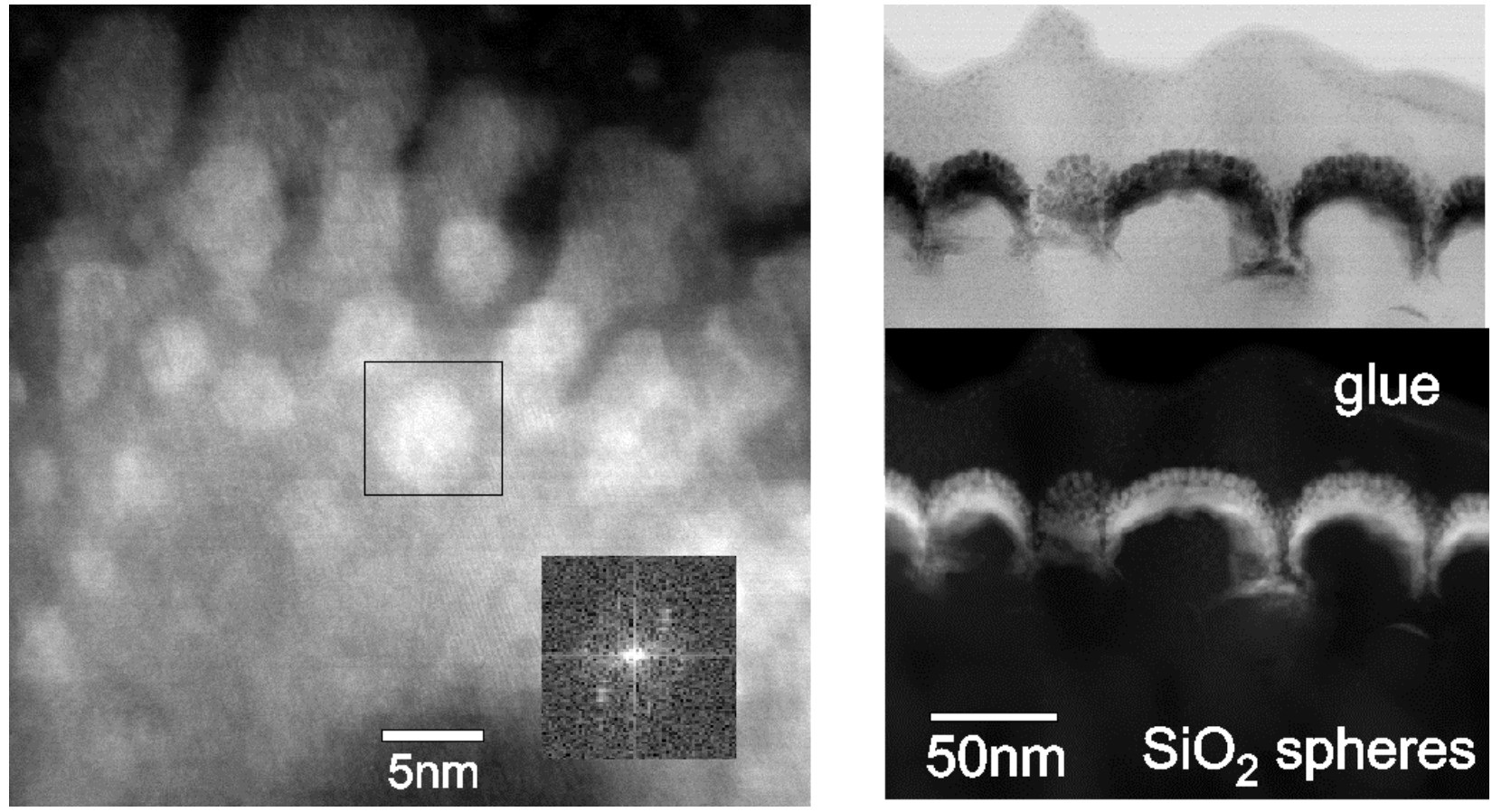

FIG.1. Bright field (upper right) and HAADF images of the hedgehog-like nanostructures, showing the distribution of high- $\mathrm{Z}$ elements $(\mathrm{Co} / \mathrm{Pt})$. The inset shows the 3 -fold symmetry of a nano-crystal.
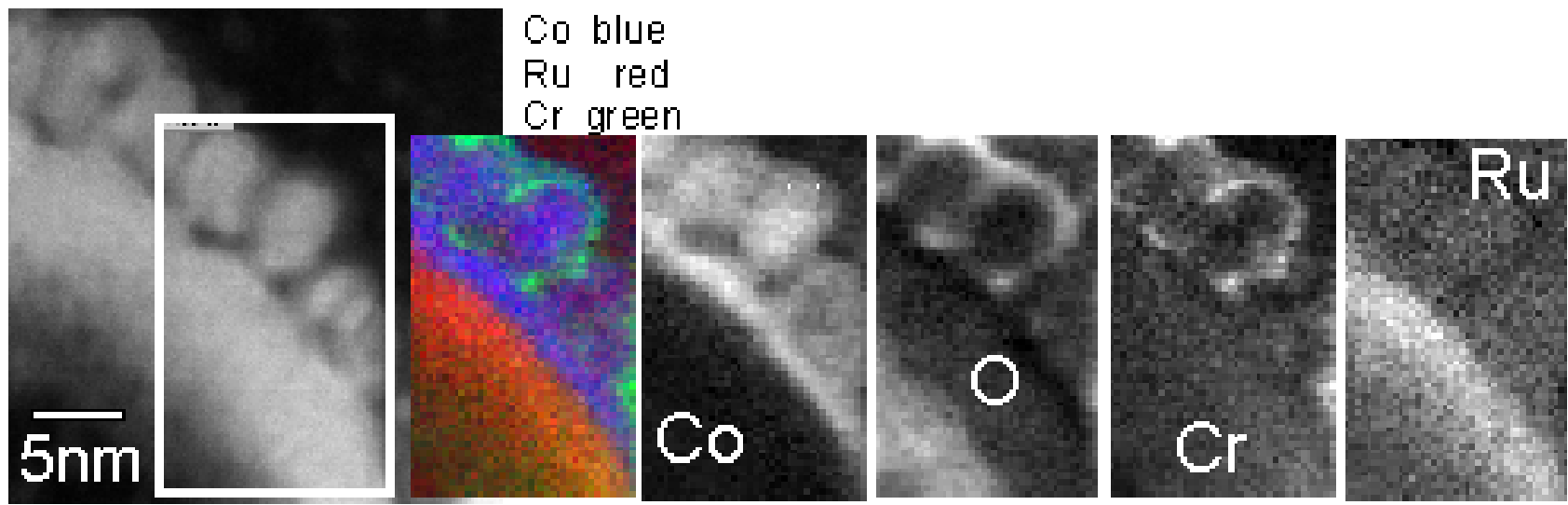

FIG. 2. Element map using EELS spectrum imaging in a NION Cs-corrected dedicated STEM.

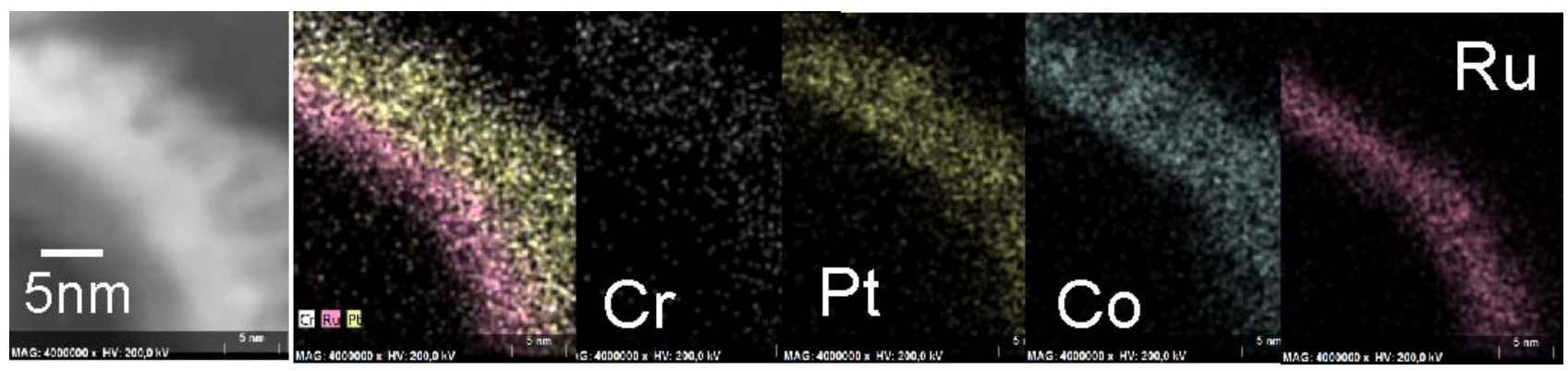

FIG. 3. HAADF and SDD-EDS map (raw data) of the same sample area in an uncorrected Jeol $\mathrm{STEM}$, the second frame shows $\mathrm{Ru}, \mathrm{Pt}, \mathrm{Cr}$. Pt appears with $\mathrm{Co} . \mathrm{Ru}$ is part of the seed layer. 